\title{
FINITE-DIMENSIONAL DESCRIPTION OF THE LONG-TERM DYNAMICS FOR THE 2D DOI-HESS MODEL FOR LIQUID CRYSTALLINE POLYMERS IN A SHEAR FLOW*
}

\author{
JESENKO VUKADINOVIC ${ }^{\dagger}$
}

\begin{abstract}
The existence of inertial manifolds for a Smoluchowski equation arising in the 2D Doi-Hess model for liquid crystalline polymers subjected to a shear flow is investigated. The presence of a non-variational drift term dramatically complicates the long-term dynamics from the variational gradient case, in which it is solely characterized by the steady states. Several transformations are used in order to transform the equation into a form suitable for application of the standard theory of inertial manifolds. A nonlinear and nonlocal transformation developed in [J. Vukadinovic, Nonlinearity, 21, 1533-1545, 2008] and [J. Vukadinovic, Commun. Math. Phys., 2008, to appear] is used to eliminate the first-order derivative from the micro-micro interaction term. A traveling wave transformation eliminates the first-order derivative from the non-variational term, transforming the equation into a nonautonomous one for which the theory of nonautonomous inertial manifolds applies.
\end{abstract}

Key words. Doi-Hess model, Smoluchowski equation, shear flow, nonautonomous inertial manifolds, Schrödinger-like equation

AMS subject classifications. $35 \mathrm{Kxx}, 70 \mathrm{Kxx}$

\section{Introduction}

One of the most prominent models for non-Newtonian fluids is the Doi-Hess molecular kinetic theory for nematic liquid crystalline polymers. Polymer molecules are viewed as identical rigid rods (cylinders) of length $L$ and diameter $b$, with $b \ll L$. A population of these molecules is described by a probability distribution function, $f(t, x, \mathbf{m})$, for the axis of symmetry $\mathbf{m} \in S^{2}$ of the molecule with the center of mass at $x$ at time $t$. Accounting for Brownian effects leading to rotational and translational diffusion, effects of the flow, and intermolecular interaction, the evolution of $f$ is governed by the so-called Smoluchowski equation

$$
\partial_{t} f+u \cdot \nabla_{x} f+\nabla_{\mathbf{m}} \cdot(W f)=D \Delta_{x} f+D_{r} \Delta_{\mathbf{m}} f+\left(D_{r} / k_{B} T\right) \nabla_{\mathbf{m}} \cdot\left(\nabla_{\mathbf{m}} V f\right),
$$

where $\nabla_{\mathbf{m}}=\mathbf{m} \times \partial_{\mathbf{m}}$ stands for the gradient operator on the unit sphere and $\Delta_{\mathbf{m}}=\nabla_{\mathbf{m}}^{2}$ stands for the Laplace-Beltrami operator. The constants $c, D_{r}, D, T$ and $k_{B}$ represent the concentration, (pre-averaged) rotational diffusivity, translational diffusivity, absolute temperature $T$, and the Boltzmann constant $k_{B}$, respectively. The equation is also often studied in two dimensions, in which case the orientations $\mathbf{m} \in S^{1}$, and the above differential operators are modified correspondingly. In the context of nematic polymers, the equation was first proposed in the works of Doi [10] and Hess [20]. It accounts for both the micro-micro interaction between the rods and the macro-micro interaction when the equation is coupled to macroscopic fluid equations. If, however, the interaction with the ambient flow is neglected, the equation is a nonlinear Fokker-Planck equation - a gradient system with the free energy as the Lyapunov functional. Historically, the Smoluchowski equation was preceded by a variational model proposed by Onsager in his seminal work [31]. Onsager calculated the free energy functional and derived the Euler-Lagrange equation for the steady-states. He

\footnotetext{
*Received: July 3, 2008; accepted (in revised version): October 11, 2008. Communicated by Pingwen Zhang.

${ }^{\dagger}$ Department of Mathematics, CUNY-College of Staten Island, 1S-208, 2800 Victory Boulevard, Staten Island, NY 10314, Tel: 718-982-3632, USA (vukadino@math.csi.cuny.edu).
} 
proposed that the interparticle (micro-micro) interaction, due to the excluded volume effects, be modelled using the mean-field ansatz

$$
V(t, x, \mathbf{m})=k_{B} T \int_{0}^{2 \pi} \beta\left(\mathbf{m}, \mathbf{m}^{\prime}\right) f\left(x, \mathbf{m}^{\prime}, t\right) d \mathbf{m}^{\prime}=\langle\beta(\mathbf{m}, \cdot)\rangle_{f(t)} .
$$

The function $\beta\left(\mathbf{m}, \mathbf{m}^{\prime}\right)$ represents a volume surrounding a molecule with orientation $\mathbf{m}$ within which the center of mass of a molecule with orientation $\mathbf{m}^{\prime}$ is not allowed. It depends on the shape of the particles, and in the case of cylindric rods it is given by the formula $\beta\left(\mathbf{m}, \mathbf{m}^{\prime}\right)=2 c d L^{2}\left|\mathbf{m} \times \mathbf{m}^{\prime}\right|$. Expanding $\beta$ in terms of products of irreducible tensors and retaining only the second term in this expansion, one arrives at the formula $\beta\left(\mathbf{m}, \mathbf{m}^{\prime}\right)=-3 / 2 U\left(\left(\mathbf{m} \cdot \mathbf{m}^{\prime}\right)^{2}-1 / 3\right)$, where $U \propto c L^{2} b$ denotes the intensity of the potential. Employing this $\beta$ instead of the one proposed by Onsager yields the so-called Maier-Saupe potential (see [29]). This significantly simplifies the mathematical treatment of the model. Nevertheless, it is widely accepted that it affords sufficient degrees of freedom to capture the essential dynamics of the micromicro interaction. In a recent development, the bifurcation diagram for the Onsager equation (and therefore also Smoluchowski equation in the absence of the flow) with the Maier-Saupe potential was confirmed rigorously (see $[6,7,9,13,25,26]$ ). The equation undergoes two bifurcations. At a lower potential intensity, the equation undergoes a saddle-node bifurcation, in which a prolate nematic branch of steady-states (where probability distribution concentrates in one direction) and an oblate nematic branch of steady-states (where the probability distribution concentrates uniformly on the equator) emerge. At a higher potential intensity, the equation undergoes a transcritical bifurcation. The oblate branch intercects with the isotropic state, and there is a transfer of stability. Since in this case the Smoluchowski equation is a gradient system, its global attractor is fairly simple; it merely consists of the steady states and their unstable manifolds.

The microscopic Smoluchowski equation is coupled to macroscopic fluid equations (e.g. Navier-Stokes equations) via the drift term

$$
W(x, \mathbf{m}, t)=(I-\mathbf{m m})\left(\mathbf{m} \cdot \nabla_{x}\right) u
$$

and via the viscoelastic stresses that the mesogenic insertions induce in the fluid. There are many challenges to the analysis of the full model, and one resorts to simplifications. Even the simplest situation of spatially homogeneous suspensions in a shear flow leads to complicated and peculiar dynamical behavior. The presence of a non-variational symmetry breaking drift term in the Smoluchowski equation dramatically complicates the dynamics. The equation ceases to be a gradient system, and the attractor becomes a very complicated set, exhibiting not only steady-states, but also various time-periodic solutions (see [12, 17, 18, 24, 27, 30], and even chaos (see $[1,19])$. Despite many existing numerical simulations, a rigorous bifurcation analysis presents a great challenge.

Although intrinsically infinite-dimensional, many dissipative parabolic systems exhibit long-term dynamics with properties typical of finite-dimensional dynamical systems. The global attractor, often considered the central object in the study of long-term behavior of dynamical systems, appears to be inadequate in capturing this finite-dimensionality, even when its Hausdorff dimension is finite. This is mainly due to two facts. Firstly, the global attractor can be a very complicated set, not necessarily a manifold. The question whether the dynamics on it can be described by a system of ODEs is yet to be resolved in the literature. Secondly, although all solutions approach 
this set, they do so at arbitrary rates, algebraic or exponential, and consequently the dynamics outside the attractor are not tracked very well on the attractor itself. When they exist, inertial manifolds emerge as the most adequate objects to capture the finite-dimensionality of a dissipative parabolic PDE. Introduced by Foias at al. in [15], they are defined to remedy the shortcomings of the global attractor just described; they should be finite dimensional positive-invariant Lipschitz manifolds which attract all solutions exponentially, and on which the solutions of the underlying PDE are recoverable from solutions of a system of ODEs, termed 'inertial form'.

The challenge to proving the existence of inertial manifolds for the Smoluchowski equation lies in the presence of the gradient in the nonlinear Fokker-Planck and the drift terms. In its original form the equation does not satisfy the spectral gap condition required in all existing theorems for proving the existence of inertial manifolds. The problem was open for a while, and the author recently succeeded in proving their existence for the gradient case by employing a nonlinear nonlocal transformation which eliminates the first-order derivatives from the equation, transforming it into a Schrödinger-like equation. The question remained open for the case in which the Smoluchowski equation is not a gradient system, and for which the question of existence of inertial manifolds becomes even more important due to the complex structure of the global attractor. In this paper, we consider the two-dimensional shear flow case. We employ a similar nonlinear nonlocal transform as the ones in papers [34, 35] to eliminate the gradient from the nonlinear Fokker-Planck term. However, we are still left with gradients in the drift term. The variational portion is then eliminated by another transform of a similar type, while the non-variational portion is eliminated using a traveling wave transformation. The trade-off is that the resulting equation contains a time-dependent forcing term, and it is therefore nonautonomous. The spectral gap condition, however, is now satisfied, and we apply the theory of nonautonomous inertial manifolds, which are obtained as time-dependent periodic sets. The theory of inertial manifolds is very well established, even for nonautonomous systems (see $[21,22,23]$ ), and there are several different methods for proving their existence, all of which yield the spectral gap condition as one of the (sufficient) conditions for their existence. In the appendix, we shall review some concepts from the theory of nonautonomous dynamical systems, such as the pullback attractor. We shall define nonautonomous inertial manifolds, and, following [2], we shall sketch the proof for their existence.

Since the global attractor is completely embedded in the inertial manifold, the dynamics on it are governed by the inertial form. This, at least theoretically at this point, provides an avenue to a rigorous bifurcation analysis of the equation, as well as significant improvement of the existing numerical studies. Some directions employ nonlinear Flocket theory, or study the equation as a nonautonomous perturbation of a gradient system.

Let us remark here that the described method still does not work for the threedimensional case in the presence of a flow. In paper [35], the three-dimensional flow in the absence of the ambient flow is treated. However, finding a transformation which eliminates the first-order derivatives from the drift term still presents a challenge.

\section{Preliminaries}

We study a Smoluchowski equation for a spatially homogeneous suspensions of rodlike polymers

$$
\partial_{t} f+\nabla_{\mathbf{m}} \cdot(W f)=D_{r} \Delta_{\mathbf{m}} f+\left(D_{r} / k_{B} T\right) \nabla_{\mathbf{m}} \cdot\left(\nabla_{\mathbf{m}} V f\right) .
$$


The interparticle (micro-micro) interaction term, due to the excluded volume effects, is given by the Maier-Saupe potential

$$
V(x, \mathbf{m}, t)=k_{B} T \int_{0}^{2 \pi} \beta\left(\mathbf{m}, \mathbf{m}^{\prime}\right) f\left(x, \mathbf{m}^{\prime}, t\right) d \mathbf{m}^{\prime}=\langle\beta(\mathbf{m}, \cdot)\rangle_{f(t)},
$$

where $\beta\left(\mathbf{m}, \mathbf{m}^{\prime}\right)=-3 / 2 U\left(\left(\mathbf{m} \cdot \mathbf{m}^{\prime}\right)^{2}-1 / 3\right) \quad($ the $3 \mathrm{D}$ case $)$ or $\beta\left(\mathbf{m}, \mathbf{m}^{\prime}\right)=-2 U((\mathbf{m}$. $\left.\mathbf{m}^{\prime}\right)^{2}-1 / 2$ ) (the $2 \mathrm{D}$ case). The macro-micro interaction term

$$
W(x, \mathbf{m}, t)=(I-\mathbf{m m})\left(\mathbf{m} \cdot \nabla_{x}\right) u
$$

is due to the rotation of the axes by the velocity gradients $\nabla_{x} u$. In this paper, we shall consider spatially homogeneous suspensions in a plane $\left(f(x, \mathbf{m}, t)=f(\mathbf{m}, t), x \in \mathbb{R}^{2}\right.$, $\left.\mathbf{m} \in S^{1}\right)$ under an imposed shear flow $u\left(x_{1}, x_{2}\right)=\left(G x_{2}, 0\right)$, where $G$ is the shear rate. We express the particle orientations using a local variable $\varphi$, i.e., $\mathbf{m}(\varphi)=(\cos \varphi, \sin \varphi)$, and write $f(\varphi)$ instead of $f(\mathbf{m})$. We will also use the notation $\mathbf{w}(\varphi)=(\cos 2 \varphi, \sin 2 \varphi)$. The simplest quantity representing the anisotropy of a probability distribution $f$ is the orientational order-parameter tensor, which is calculated as the traceless equivalent of the second moment tensor

$$
\mathcal{S}[f]=\langle\mathbf{m m}-\mathrm{I} / 2\rangle_{f}=\int_{0}^{2 \pi}[\mathbf{m}(\varphi) \mathbf{m}(\varphi)-\mathrm{I} / 2] f(\varphi) d \varphi .
$$

The scalar order parameter

$$
S[f]=(2 \mathcal{S}[f]: \mathcal{S}[f])^{\frac{1}{2}}=\left(\langle\mathbf{w}\rangle_{f} \cdot\langle\mathbf{w}\rangle_{f}\right)^{\frac{1}{2}} \in[0,1]
$$

represents the degree of molecular alignment. For the isotropic phase, $\bar{f}=1 / 2 \pi, S[\bar{f}]=$ 0 , and for the perfect alignment $S[f]=1$.

After rescaling, the Smoluchowski equation becomes

$$
f_{t}=f_{\varphi \varphi}+(W f)_{\varphi}+\left(V_{\varphi} f\right)_{\varphi}
$$

where the Maier-Saupe potential is given by

$$
V[f]=-\frac{U}{2}(\mathbf{m m}-\mathrm{I} / 2): \mathcal{S}[f]=-U\langle\mathbf{w}\rangle_{f} \cdot \mathbf{w} .
$$

Here we use the notation $\langle g\rangle_{f}=\int_{0}^{2 \pi} f(\varphi) g(\varphi) d \varphi$. Observe that $|V[f]| \leq U$. Also,

$$
W(\varphi)=G \sin ^{2} \varphi=\frac{G}{2}(1-\cos 2 \varphi) .
$$

Denoting

$$
\tilde{V}(\varphi)=-\frac{G}{4} \sin 2 \varphi
$$

equation (2.1) can be written as

$$
f_{t}-\frac{G}{2} f_{\varphi}=f_{\varphi \varphi}+\left(\left(V_{\varphi}+\tilde{V}_{\varphi}\right) f\right)_{\varphi}
$$

With regard to the existence, uniqueness and regularity of solutions of (2.1), it is easy to prove the following theorem (see $[6,7]$ ). 
THEOREM 2.1. Let $f_{0}>0$ be a continuous function on $S^{1}$ such that $\int_{0}^{2 \pi} f_{0}=1$. A unique smooth solution $f(t)=\Sigma(t) f_{0}$ of (2.1) for an initial data $f(0)=f_{0}$ exists for all nonnegative times, and remains positive and normalized

$$
\int_{0}^{2 \pi} f(\varphi, t) d \varphi=1
$$

Symmetry with respect to the origin - reflecting the fact that that we do not distinguish between orientations $\mathbf{w}$ and $-\mathbf{w}-$ is preserved. Therefore, we can expend the solutions in Fourier series as

$$
f(\varphi, t)=\frac{1}{2 \pi}+\frac{1}{\pi} \sum_{k=1}^{\infty}\left[a_{k}(t) \cos (2 k \varphi)+b_{k}(t) \sin (2 k \varphi)\right],
$$

where

$$
\begin{aligned}
& a_{k}(t)=\langle\cos (2 k \varphi)\rangle_{f(t)}=\int_{0}^{2 \pi} \cos (2 k \varphi) f(\varphi, t) d \varphi, \\
& b_{k}(t)=\langle\sin (2 k \varphi)\rangle_{f(t)}=\int_{0}^{2 \pi} \sin (2 k \varphi) f(\varphi, t) d \varphi .
\end{aligned}
$$

In this setting, the 2D Smoluchowski equation can also be written as an infinite system of ODEs

$$
\begin{aligned}
a_{0}= & 1 \\
b_{0}= & 0 \\
a_{k}^{\prime}= & -4 k^{2} a_{k}+2 U k\left[a_{1}\left(a_{k-1}-a_{k+1}\right)-b_{1}\left(b_{k-1}+b_{k+1}\right)\right] \\
& +\frac{k}{2} G\left(-b_{k-1}+2 b_{k}-b_{k+1}\right) \\
b_{k}^{\prime}= & -4 k^{2} b_{k}+2 U k\left[b_{1}\left(a_{k-1}+a_{k+1}\right)+a_{1}\left(b_{k-1}-b_{k+1}\right)\right] \\
& -\frac{k}{2} G\left(-a_{k-1}+2 a_{k}-a_{k+1}\right) .
\end{aligned}
$$

Multiplying the equation for $a_{k}^{\prime}$ by $a_{k} / 2$ and the equation for $b_{k}^{\prime}$ by $b_{k} / 2$, and adding the two over $k=1,2,3, \ldots$ implies the following equation

$$
\frac{1}{2} \frac{d}{d t} \sum_{k=1}^{\infty} \frac{a_{k}^{2}+b_{k}^{2}}{k}+4 \sum_{k=1}^{\infty} k\left(a_{k}^{2}+b_{k}^{2}\right)=2 U\left(a_{1}^{2}+b_{1}^{2}\right)+\frac{G}{2} b_{1} .
$$

This implies that the equation is dissipative in the space $H^{-1 / 2}\left(S^{1}\right)$, and also the fact that if $S[f(t)] \rightarrow 0$ as $t \rightarrow \infty$, then $f(t) \rightarrow \frac{1}{2 \pi}$ as $t \rightarrow \infty$. Similar to [8], one can prove the dissipativity in Gevrey classes of functions, which, in turn, imply the dissipativity in any Sobolev space $H^{k}\left(S^{1}\right)$. Also, the fact that $a_{1}$ and $b_{1}$ are determining modes is proven in a similar fashion. Let us state the following:

TheOREM 2.2. Equation (2.1) is dissipative in the Sobolev space $H^{k}\left(S^{1}\right)$, for any $k \in \mathbb{N}_{0}$, in the following sense. There exists $\rho_{k}=\rho_{k}(U)>0$ such that for any bounded set $\mathcal{U} \subset L^{2}\left(S^{1}\right)$, there exists $T_{\mathcal{U}}>0$ so that for positive $f_{0} \in \mathcal{U}$ and $t \geq T_{\mathcal{U}}$ the solution $f(t)=\Sigma(t) f_{0}$ satisfies $\left\|\partial_{\varphi}^{k} f(t)\right\|_{L^{2}} \leq \rho_{k}$. In other words, the ball $B_{\rho_{k}}^{k}=\left\{f \in H^{k}\left(S^{1}\right)\right.$ : $\left.\|f\|_{H^{k}} \leq \rho_{k}\right\}$ is an absorbing set: all solutions of (2.1) enter this set to remain there, 
eventually. In particular, the solution operator $\Sigma(t)$ is compact, and the equation has a finite-dimensional global attractor $\mathcal{A}$. This is the maximal compact set which is invariant: $\Sigma(t) \mathcal{A}=\mathcal{A}$ for all $t \in \mathbb{R}$, and attracts all solutions: $\operatorname{dist}\left(\Sigma(t) f_{0}, \mathcal{A}\right) \rightarrow 0$ as $t \rightarrow \infty$ for any $f_{0} \in L^{2}\left(S^{1}\right)$.

Let us also remark that the scalar order parameter tensor evolves according to the equation

$$
\begin{aligned}
\frac{1}{2} \frac{d}{d t} S[f(t)]^{2}= & (2 U-4) S[f(t)]^{2} \\
& +2 U\left(-a_{1}^{2} a_{2}-2 a_{1} b_{1} b_{2}+b_{1}^{2} a_{2}\right)+\frac{G}{2}\left(b_{1}-a_{1} b_{2}+a_{2} b_{1}\right) .
\end{aligned}
$$

We shall also use the fact that when $U>2$ there exists $s_{U}>0$ such that for any solution $f(t)$ of equation (2.1) there exists a time $T_{f}>0$ so that $S[f(t)]>s_{U}$ for all $t \geq T_{f}$.

\section{The main result}

3.1. Schrödinger-like equation. Let us begin this section by explaining the method behind transforming the Smoluchowski equation into a form suitable for the application of the classical theory of inertial manifolds. The idea originates in the theory for linear Fokker-Planck equations of the form

$$
\partial_{t} \psi=\Delta \psi+\nabla \cdot(\psi \nabla V)
$$

where $V$ is a given (possibly time-dependent) potential. Even for this linear equation, the classical theory of inertial manifolds generally does not apply, since the spectral gap condition is not satisfied. However, the transform

$$
\psi=u e^{-V / 2}
$$

transforms the Fokker-Planck equation into what is referred to as a Schrödinger-like equation

$$
\partial_{t} u=\Delta u+\frac{1}{2}\left[\partial_{t} V+\Delta V-\frac{1}{2}|\nabla V|^{2}\right] u .
$$

As long as the laplacian operator has spectral gaps which are increasing indefinitely, after imposing some conditions on $V$ it can be proven that this Schrödinger-like equation possesses inertial manifolds. Due to the fact that the above transformation is invertible, we can infer the existence of inertial manifolds for the linear Fokker-Planck equation as well.

The situation is more complicated for the Smoluchowski Equation (2.1) since the potential is not given a priori. Rather, it is related in a non-local fashion to the probability function $f$

$$
V[f]=-U\langle\mathbf{w}\rangle_{f} \cdot \mathbf{w}
$$

As a projection, this relation is not invertible. Following the discussion for the linear Fokker-Planck equation, we introduce the transform

$$
u=f e^{V / 2}=f e^{-\frac{U}{2}\langle\mathbf{w}\rangle_{f} \cdot \mathbf{w}}
$$

which is now a nonlinear nonlocal transformation. Similar to above, substitution into the Smoluchowski equation yields the equation

$$
u_{t}=u_{\varphi \varphi}+(W u)_{\varphi}+\frac{1}{2}\left(V_{t}+V_{\varphi \varphi}-\frac{1}{2}\left(V_{\varphi}\right)^{2}-W V_{\varphi}\right) u
$$


The question immediately arises whether this equation can be viewed as a closed equation in $u$, or, in other words, whether $V$ (and therefore by virtue of (3.1) $f$, as well) can be retrieved from $u$. Unfortunately, this seems not to be the case. However, it turns out that the following, very similar transformation

$$
u=f e^{-V / 2}=f e^{\frac{U}{2}\langle\mathbf{w}\rangle_{f} \cdot \mathbf{w}}
$$

is invertible on a large open set of functions. The reason is that, given a function $u$, one can exploit the conservation of the $L^{1}$ norm to first recover the second eigenmode $\langle\mathbf{w}\rangle_{f} \cdot \mathbf{w}$, thus also $V[f]$ and $f$ from $u$. The change of the sign in the exponent of the transformation is easily accomplished if the transformation (3.2) is preceded by a transformation which changes the sign of the second eigenmode in the Fourier expansion of $f$ and preserves the positivity of $f$, for example

$$
g=\Theta(f)=f-2 P_{2} f+d=f+c V+d,
$$

where $c=\frac{2}{U \pi}$ and $d=\frac{4}{\pi}$. Notice that $f>0$ implies $g>0$ and $\int_{0}^{2 \pi} f(\varphi) d \varphi=1$ implies $\int_{0}^{2 \pi} g(\varphi) d \varphi=9$. It can be easily seen that $f$ satisfies the Smoluchowski equation if and only if $g$ satisfies

$$
g_{t}=g_{\varphi \varphi}+(W g)_{\varphi}+\left(V_{\varphi} g\right)_{\varphi}+H(g, \varphi)
$$

where

$$
H(g, \varphi)=c\left[V_{t}-V_{\varphi \varphi}-\left(V\left(W+V_{\varphi}\right)\right)_{\varphi}\right]-d\left(W+V_{\varphi}\right)_{\varphi} .
$$

As already indicated, the transformation

$$
u=g \exp (V / 2)=g e^{\frac{U}{2}\langle\mathbf{w}\rangle_{g} \cdot \mathbf{w}}
$$

can be proven to be invertible, and it eliminates the first-order derivative from the nonlinearity. This will be done in the next section by employing a Laplace-like transform. Note that from now on,

$$
V[g]=U\langle\mathbf{w}\rangle_{g} \cdot \mathbf{w} .
$$

One can now easily verify that the function $g$ satisfies (2.1) if and only if $u=g \exp (V / 2)$ satisfies the equation

$$
u_{t}=u_{\varphi \varphi}+(W u)_{\varphi}+\frac{1}{2}\left(V_{t}+V_{\varphi \varphi}-\frac{1}{2}\left(V_{\varphi}\right)^{2}-W V_{\varphi}\right) u+H(g) e^{V / 2}
$$

In view of the equation for the evolution of $V[g]$ and the fact that $\|V[g]\|_{\infty} \leq U$, we can write the latter equation as

$$
u_{t}=u_{\varphi \varphi}+(W u)_{\varphi}+F(g, \varphi)
$$

where $F$ depends Lipschitz-continuously on $g$ and $\varphi$ and is periodic in $\varphi$. Our next goal is to express $g$ as a function of $u$ in order to view (3.6) as a closed semilinear parabolic equation in $u$. 
3.2. Transformation inverse. Let us rewrite the transform (3.4) in the following form

$$
g=u e^{-V / 2}=u e^{-\frac{U}{2}\langle\mathbf{w}\rangle_{g} \cdot \mathbf{w}} .
$$

Given $u$, this is a nonlinear nonlocal equation in $g$. The conservation of the $L^{1}$ norm yields that

$$
\int_{0}^{2 \pi} u(\varphi) e^{-(U / 2)\langle\mathbf{w}\rangle_{g} \cdot \mathbf{w}(\varphi)} d \varphi=9
$$

and multiplication by $\mathbf{w}$ and integration yields the equation

$$
\int_{0}^{2 \pi} u(\varphi) \mathbf{w}(\varphi) e^{-(U / 2)\langle\mathbf{w}\rangle_{g} \cdot \mathbf{w}(\varphi)} d \varphi=\langle\mathbf{w}\rangle_{g}
$$

It turns out that these two equations are sufficient to solve for $g$. For any $u \in L^{1}\left(S^{1}\right)$, we define the Laplace-like transform $\widehat{u} \in C^{\infty}\left(\mathbb{R}^{2}\right)$

$$
\widehat{u}(\mathbf{x})=\int_{0}^{2 \pi} u(\varphi) e^{-\mathbf{x} \cdot \mathbf{w}(\varphi)} d \varphi
$$

Equation (3.7) becomes

$$
\widehat{u}\left((U / 2)\langle\mathbf{w}\rangle_{g}\right)=9
$$

and equation (3.8) becomes

$$
-\nabla \widehat{u}\left((U / 2)\langle\mathbf{w}\rangle_{g}\right)=\langle\mathbf{w}\rangle_{g} .
$$

In order to solve the latter two equations for $\langle\mathbf{w}\rangle_{g}$, let us develop the following framework. Firstly, similar to the Fourier and the Laplace transforms, for $\mathbf{a} \in \mathbb{R}^{2}$, we define the operator

$$
\mu_{\mathbf{a}} u(\varphi):=u(\varphi) e^{-\mathbf{a} \cdot \mathbf{w}(\varphi)} \in L^{1}\left(S^{1}\right)
$$

and so

$$
\widehat{\mu_{\mathbf{a}} u}(\mathbf{x})=\int_{0}^{2 \pi} u(\varphi) e^{-(\mathbf{x}+\mathbf{a}) \cdot \mathbf{w}(\varphi)} d \varphi=: \tau_{\mathbf{a}} \widehat{u}(\mathbf{x}) .
$$

We define the function sets $\mathcal{H}=L^{2}\left(S^{1} ; \mathbb{R}^{+}\right)$, and

$$
X=\left\{g \in \mathcal{H}: \int_{0}^{2 \pi} g(\varphi) d \varphi=9\right\} .
$$

Also let

$$
\mathcal{X}=\left\{u \in \mathcal{H}: \int_{0}^{2 \pi} \mu_{\mathbf{a}} u(\varphi) d \varphi<9 \text { for some } \mathbf{a} \in \mathbb{R}^{2}\right\},
$$

$\mathcal{X}_{1}=\left\{u \in \mathcal{X}: \int_{0}^{2 \pi} u(\varphi) d \varphi \geq 9\right\}$, and $\mathcal{X}_{2}=\left\{u \in \mathcal{X}: \int_{0}^{2 \pi} u(\varphi) d \varphi<9\right\}$, so that $\mathcal{X}=\mathcal{X}_{1} \cup$ $\mathcal{X}_{2}$. For $u \in \mathcal{X}$ we have

$$
\begin{array}{r}
\nabla \widehat{u}(\mathbf{x})=-\int_{0}^{2 \pi} \mu_{\mathbf{x}} u(\varphi) \mathbf{w}(\varphi) d \varphi \\
\nabla \nabla \widehat{u}(\mathbf{x})=\int_{0}^{2 \pi} \mu_{\mathbf{x}} u(\varphi)(\mathbf{w}(\varphi) \mathbf{w}(\varphi)) d \varphi
\end{array}
$$


$\nabla \nabla \widehat{u}(\mathbf{x})$ is positive definite, since by Cauchy-Schwarz

$$
\operatorname{det}(\nabla \nabla \widehat{u})=\left\langle w_{1}^{2}\right\rangle\left\langle w_{2}^{2}\right\rangle-\left\langle w_{1} w_{2}\right\rangle^{2}>0,
$$

where $\langle f\rangle=\int_{0}^{2 \pi} f(\varphi) \mu_{x} u(\varphi) d \varphi$, so $\widehat{u}$ is a concave.

If $u \in \mathcal{X}_{1}$, the level set

$$
\Gamma(u)=\left\{\mathbf{x} \in \mathbb{R}^{2} \mid \widehat{u}(\mathbf{x}) \leq 9\right\}
$$

is a nonempty convex set, and the there exists a unique point $\mathbf{r} \in \partial \Gamma(u)$ so that $|\mathbf{r}|=\operatorname{dist}(\Gamma(u), \mathbf{o})$, where $\mathbf{o}=(0,0)$. Note that $\mathbf{r}$ is the unique point on $\partial \Gamma(u)$ for which there exists $U>0$ such that

$$
\nabla \widehat{u}(\mathbf{r})=-\frac{2}{U} \mathbf{r}
$$

We now define the mappings

$$
\begin{aligned}
R: \mathcal{X}_{1} & \rightarrow \mathbb{R}^{2}, \\
u & \mapsto \mathbf{r}, \\
\mathcal{G}: \mathcal{X}_{1} & \rightarrow X, \\
u & \mapsto g=\mu_{R(u)} u=u e^{-R(u) \cdot \mathbf{w},} \\
Y: \mathcal{X}_{1} & \rightarrow \mathbb{R}^{2}, \\
u & \mapsto-(\nabla \widehat{u})(R(u))=\int_{0}^{2 \pi} u(\varphi) e^{-R(u) \cdot \mathbf{w}(\varphi)} \mathbf{w}(\varphi) d \varphi=<\mathbf{w}>_{\mathcal{G}(u)} \\
\Upsilon: \mathcal{X}_{1} & \rightarrow \mathbb{R}^{+}, \\
u & \mapsto U=2|R(u)| /|Y(u)| .
\end{aligned}
$$

Note the inequality $R(u) \leq \Upsilon(u) / 2$. We will need the following:

Lemma 3.1. $R, \mathcal{G}, Y$, and $\Upsilon$ are continuous functions on $\mathcal{X}_{1}$.

We prove the continuity of $R$, and the continuities of $\mathcal{G}, Y$ and $\Upsilon$ follow. To prove the statement by contradiction, we chose a sequence $\left(v_{n}\right)_{n \in \mathbb{N}}$ in $\mathcal{X}_{1}$ and $u \in \mathcal{X}_{1}$ such that $v_{n} \rightarrow u$ in $L^{2}\left(S^{2}\right)$. This obviously implies $\widehat{v_{n}} \rightarrow \widehat{u}$ and ${\widehat{v_{n}}}^{\prime} \rightarrow \widehat{u}^{\prime}$ in $L^{\infty}\left(S^{2}\right)$. Let $\mathbf{r}=R(u), \mathbf{s}_{n}=R\left(v_{n}\right)$, and suppose $\mathbf{s}_{n} \nrightarrow \mathbf{r}$ as $n \rightarrow \infty$. Let $U_{n}=2\left|R\left(v_{n}\right)\right| /\left|Y\left(v_{n}\right)\right|=$ $4\left|\mathbf{s}_{n}\right| /\left|\nabla \widehat{v}_{n}\left(\mathbf{s}_{n}\right)\right|$. One can easily observe that the sequence $\left(\mathbf{s}_{n}\right)$ is bounded. Therefore, without loss of generality, we can assume that $\mathbf{s}_{n} \rightarrow \mathbf{s} \neq \mathbf{r}$ as $n \rightarrow \infty$. Because of the convergence in the sup norm, $\widehat{v_{n}}\left(\mathbf{s}_{n}\right) \rightarrow \widehat{u}(\mathbf{s})$ and $\nabla \widehat{v_{n}}\left(\mathbf{s}_{n}\right) \rightarrow \nabla \widehat{u}(\mathbf{s})$. Therefore, $\widehat{v_{n}}\left(\mathbf{s}_{n}\right)=9$ implies $\widehat{u}(\mathbf{s})=9$, and $U_{n} \rightarrow U:=2|\mathbf{s}| /|\nabla \widehat{u}(\mathbf{s})|$, so $\nabla \widehat{u}(\mathbf{s})=-\frac{2}{U} \mathbf{s}$. This is a contradiction to $\mathbf{s} \neq \mathbf{r}$.

As discussed earlier, $g(t)=\Theta(f(t))$ is a solution of (3.3) for some $U>0$ if and only if

$$
u(t)=g(t) e^{V[g(t)] / 2}
$$

satisfies

$$
u_{t}=u_{\varphi \varphi}+(W u)_{\varphi}+F(\mathcal{G}(u), \varphi) .
$$

As already mentioned, we need Lipschitz continuity of the nonlinear term in order to apply the classical theory of inertial manifolds. In the following lemmas, we shall establish some facts about the Lipschitz continuity of the transformation. 
Lemma 3.2. Let $U>0$ be fixed, and let $\mathcal{X}_{U}=\Upsilon^{-1}\{U\}$. The functions $\left.R\right|_{\mathcal{X}_{U}},\left.\mathcal{G}\right|_{\mathcal{X}_{U}}$, and $\left.Y\right|_{\mathcal{X}_{U}}$, are Lipschitz continuous. In particular, $\mathcal{G}_{U}=\left.\mathcal{G}\right|_{\mathcal{X}_{U}}: \mathcal{X}_{U} \rightarrow X$ is a Lipschitz homeomorphism. Its inverse is given by

$$
\mathcal{G}_{U}^{-1}(g)=g e^{(U / 2)<\mathbf{w}>_{g} \cdot \mathbf{w}} .
$$

We prove the Lipschitz continuity of $R$, and the others follow. Let $u, v \in \mathcal{X}_{U}$, and let $\mathbf{r}=R(u), \mathbf{s}=R(v)$. The mean-value theorem implies the existence of $\theta_{1} \in[0,1]$ and $\theta_{2} \in[0,1]$ so that, with the convexity of $\widehat{u}$ and $\widehat{v}$, we have

$$
\widehat{u}(\mathbf{s})-\widehat{u}(\mathbf{r})=\nabla \widehat{u}\left(\mathbf{r}+\theta_{1}(\mathbf{s}-\mathbf{r})\right) \cdot(\mathbf{s}-\mathbf{r}) \geq \nabla \widehat{u}(\mathbf{r}) \cdot(\mathbf{s}-\mathbf{r})=-\frac{2}{U} \mathbf{r}(\mathbf{s}-\mathbf{r})
$$

and

$$
\widehat{v}(\mathbf{r})-\widehat{v}(\mathbf{s})=\nabla \widehat{v}\left(\mathbf{s}+\theta_{2}(\mathbf{r}-\mathbf{s})\right) \cdot(\mathbf{r}-\mathbf{s}) \geq \nabla \widehat{v}(\mathbf{s}) \cdot(\mathbf{r}-\mathbf{s})=-\frac{2}{U} \mathbf{s}(\mathbf{r}-\mathbf{s}) .
$$

Adding both equations yields

$$
\int_{0}^{2 \pi}(u(\varphi)-v(\varphi))\left(e^{-\mathbf{s} \cdot \mathbf{w}(\varphi)}-e^{-\mathbf{r} \cdot \mathbf{w}(\varphi)}\right) d \varphi \geq \frac{2}{U}|\mathbf{s}-\mathbf{r}|^{2}
$$

and therefore there exists $C_{3}=C_{3}(U)>0$ so that

$$
\frac{2}{U}|\mathbf{s}-\mathbf{r}|^{2} \leq C_{3}\|u-v\|_{L^{2}}|\mathbf{r}-\mathbf{s}|
$$

and so $|R(u)-R(v)| \leq \frac{U C_{3}}{2}\|u-v\|_{L^{2}}$.

For $\kappa>0$ let us define the ball $\mathcal{B}_{\kappa}=\left\{u \in \mathcal{H}:\|u\|_{L^{2}} \leq \kappa\right\}$. Let us now choose $\kappa: \mathbb{R}^{+} \rightarrow \mathbb{R}^{+}$, continuous and increasing, such that the ball $\mathcal{B}_{\kappa(U)}$ satisfies $\mathcal{B}_{\kappa(U)} \supset$ $\mathcal{G}_{U}^{-1}\left(\Theta\left(B_{\rho_{0}(U)}\right)\right)$. Observe that the ball $\mathcal{B}_{\kappa(U)}$ is an absorbing ball in $L^{2}\left(S^{1}\right)$ for the transformed equation (3.9).

We have proved the Lipschitz continuity of the transformation on the set of functions $\Upsilon^{-1}\{U\}$ on which $\Upsilon(u)$ is kept fixed at $U$. Since the potential intensity $U$ is given a priori and it is not changed by the original equation, this is also true for the transformed equation. However, the transform changes the geometry of the phase space, and $\Upsilon^{-1}\{U\}$ is not a Hilbert space. In order to apply the classical theory, we need to imbed this set in a larger Hilbert space, and we accomplish this by allowing $U$ to change. $\Upsilon(u)$ becomes a quantity that is preserved under the solution operator. In the following lemma we expand the already established Lipschitz continuity to this larger Hilbert space.

LEMMA 3.3. Let $U_{1}>0$ and $K>0 . \quad$ Let $\mathcal{U}=\mathcal{B}_{\kappa\left(U_{1}\right)} \cap \Upsilon^{-1}\left(0, U_{1}\right) \cap\left\{u \in \mathcal{X}_{1}: K<\right.$ $|Y(u)|\}$. Then $\left.R\right|_{\mathcal{U}},\left.\mathcal{G}\right|_{\mathcal{U}},\left.Y\right|_{\mathcal{U}},\left.U\right|_{\mathcal{U}}$ are Lipschitz continuous.

Let $u, v \in \mathcal{U}$, and let $\mathbf{r}=R(u), \mathbf{s}=R(v)$. As before, we have

$$
\widehat{u}(\mathbf{s})-\widehat{u}(\mathbf{r}) \geq \frac{2}{\Upsilon(u)} \mathbf{r}(\mathbf{r}-\mathbf{s})
$$

and

$$
\widehat{v}(\mathbf{r})-\widehat{v}(\mathbf{s}) \geq \frac{2}{\Upsilon(v)} \mathbf{s}(\mathbf{s}-\mathbf{r})
$$


Since $\mathbf{r}(\mathbf{r}-\mathbf{s})+\mathbf{s}(\mathbf{s}-\mathbf{r})=|\mathbf{r}-\mathbf{s}|^{2} \geq 0$, we distinguish between the following cases:

Case 1: $\mathbf{r}(\mathbf{r}-\mathbf{s}) \geq 0$ and $\mathbf{s}(\mathbf{s}-\mathbf{r}) \geq 0$.

In this case, similar to the previous Lemma, we have

$$
\int_{0}^{2 \pi}(u(\varphi)-v(\varphi))\left(e^{-\mathbf{s} \cdot \mathbf{w}(\varphi)}-e^{-\mathbf{r} \cdot \mathbf{w}(\varphi)}\right) d \varphi \geq \frac{2}{U_{1}}|\mathbf{s}-\mathbf{r}|^{2},
$$

so $|R(u)-R(v)| \leq \frac{U_{1} C_{3}\left(U_{1}\right)}{2}\|u-v\|_{L^{2}}$.

Case 2: $\mathbf{r}(\mathbf{r}-\mathbf{s})<0$ and $\mathbf{s} \notin \Gamma(u)$.

In this case,

$$
\widehat{u}(\mathbf{s})-\widehat{u}(\mathbf{r})>0>\frac{2}{\Upsilon(v)} \mathbf{r}(\mathbf{r}-\mathbf{s})
$$

and

$$
\widehat{v}(\mathbf{r})-\widehat{v}(\mathbf{s}) \geq \frac{2}{\Upsilon(v)} \mathbf{s}(\mathbf{s}-\mathbf{r}),
$$

and one arrives at the same conclusion as in the previous case.

Case 3: $\mathbf{r}(\mathbf{r}-\mathbf{s})<0$ and $\mathbf{s} \in \Gamma(u)$.

Since $\mathbf{s} \in \Gamma(u)$, there exists $\mathbf{s}^{\prime} \in \partial \Gamma(u) \cap[\mathbf{o}, \mathbf{s}]$. Let $v^{\prime}=\mu_{\mathbf{s}-\mathbf{s}^{\prime}} v$, and so $\widehat{v}^{\prime}=\tau_{\mathbf{s}-\mathbf{s}^{\prime}} \widehat{v}$. Thus, $\widehat{v}^{\prime}\left(\mathbf{s}^{\prime}\right)=\widehat{v}(\mathbf{s})=9$, so $R\left(v^{\prime}\right)=\mathbf{s}^{\prime}$ follows. Another easy observation is that $\mathbf{r}\left(\mathbf{r}-\mathbf{s}^{\prime}\right) \leq 0$, and so

$$
\widehat{u}\left(\mathbf{s}^{\prime}\right)-\widehat{u}(\mathbf{r})=0 \geq \frac{2}{\Upsilon(v)} \mathbf{r}\left(\mathbf{r}-\mathbf{s}^{\prime}\right)
$$

and

$$
\widehat{v}^{\prime}(\mathbf{r})-\widehat{v}^{\prime}\left(\mathbf{s}^{\prime}\right) \geq \frac{2}{\Upsilon\left(v^{\prime}\right)} \mathbf{s}^{\prime}\left(\mathbf{s}^{\prime}-\mathbf{r}\right) \geq \frac{2}{\Upsilon(v)} \mathbf{s}^{\prime}\left(\mathbf{s}^{\prime}-\mathbf{r}\right),
$$

and we again conclude that $\left|\mathbf{r}-\mathbf{s}^{\prime}\right| \leq \frac{U_{1} C_{3}\left(U_{1}\right)}{2}\left\|u-v^{\prime}\right\|_{L^{2}}$. On the other hand, since $\mathbf{s}$ and $\mathbf{s}^{\prime}$ are collinear,

$$
\widehat{v}\left(\mathbf{s}^{\prime}\right)-\widehat{v}(\mathbf{s}) \geq \frac{2}{\Upsilon(v)} \mathbf{s}\left(\mathbf{s}-\mathbf{s}^{\prime}\right)=\frac{2}{\Upsilon(v)}|\mathbf{s}|\left|\mathbf{s}-\mathbf{s}^{\prime}\right| \geq K\left|\mathbf{s}-\mathbf{s}^{\prime}\right| .
$$

Since $\widehat{v}(\mathbf{s})=\widehat{u}\left(\mathbf{s}^{\prime}\right)$, we have $\left|\mathbf{s}-\mathbf{s}^{\prime}\right| \leq \frac{U_{1} C_{3}\left(U_{1}\right)}{K}\|v-u\|_{L^{2}}$. The desired result follows with the estimate

$$
\begin{aligned}
\left\|v-v^{\prime}\right\|_{L^{2}}^{2} & =\int_{0}^{2 \pi}\left(v(\varphi)-v^{\prime}(\varphi)\right)^{2} d \varphi=\int_{0}^{2 \pi} v(\varphi)^{2}\left(1-e^{\left(\mathbf{s}^{\prime}-\mathbf{s}\right) \cdot \mathbf{w}(\varphi)}\right)^{2} d \varphi \\
& =\int_{0}^{2 \pi}[\mathcal{G}(v)(\varphi)]^{2}\left(e^{\mathbf{s} \cdot \mathbf{w}(\varphi)}-e^{\mathbf{s}^{\prime} \cdot \mathbf{w}(\varphi)}\right)^{2} d \varphi \leq C_{4}^{2}\left|\mathbf{s}-\mathbf{s}^{\prime}\right|^{2}\|\mathcal{G}(v)\|_{L^{2}}^{2} \leq C_{5}^{2}\|v-u\|_{L^{2}}^{2}
\end{aligned}
$$

where the constants $C_{4}$ and $C_{5}$ depend on $K$ and $U_{1}$ only.

Case 4: $\mathbf{s}(\mathbf{s}-\mathbf{r})<0$.

The inequalities for this case follow in an analogous fashion to the previous two cases. 
Lemma 3.4. Let $0<U<U_{1}$ and $K=s_{U}$, and let $\mathcal{U}$ be defined as in the previous lemma. Let $\mathcal{V}=\mathcal{U} \cup\left(\mathcal{X}_{U} \cap \mathcal{B}_{\kappa(U)}\right)$. Then the functions $\left.R\right|_{\mathcal{V}},\left.\mathcal{G}\right|_{\mathcal{V}},\left.Y\right|_{\mathcal{V}}$, and $\left.U\right|_{\mathcal{V}}$ are Lipschitz continuous.

Let us partition $\mathcal{V}$ into three regions: $\mathcal{V}_{1}=\mathcal{X}_{U} \cap \mathcal{B}_{\kappa(U)} \cap R^{-1}\left(0, s_{1} / 2\right], \mathcal{V}_{2}=\mathcal{X}_{U} \cap$ $\mathcal{B}_{\kappa(U)} \cap R^{-1}\left(s_{1} / 2, s_{1}\right)$, and $\mathcal{U}$. By the previous two lemmas, the functions are Lipschitz continuous on any of these three regions, as well as on the sets $\mathcal{V}_{1} \cup \mathcal{V}_{2}$ and $\mathcal{V}_{2} \cup \mathcal{U}$. The Lipschitz continuity on $\mathcal{V}_{1} \cup \mathcal{U}$ follows since both of these sets are bounded in $L^{2}$ and $\operatorname{dist}\left(R\left(\mathcal{V}_{1}\right), R(\mathcal{U})\right)>s_{1} / 2$. This implies the Lipschitz continuity on $\mathcal{V}$.

3.3. Prepared equation. Before we can prepare the equation, and apply the theory, we need to preform a few more transformations in order to have the equation in the form to which the classical theory applies. Firstly, we eliminate the variational portion of the drift $\tilde{V}=-\frac{G}{4} \sin 2 \varphi$ term by employing the Lipschitz-homeomorphism $u=\mathcal{K}(v)=v e^{-\tilde{V} / 2}$. This leads similarly as before to an equation of the form

$$
v_{t}=v_{\varphi \varphi}+\frac{G}{2} v_{\varphi}+\tilde{F}(\mathcal{G}(\mathcal{K}(v)), \varphi)
$$

where $\tilde{F}$ is Lipschitz continuous in both components and is periodic in $\varphi$. Denoting

$$
N(v, \varphi)=\tilde{F}(\mathcal{G}(\mathcal{K}(v), \varphi),
$$

we can write the equation as

$$
v_{t}=v_{\varphi \varphi}+\frac{G}{2} v_{\varphi}+N(v, \varphi)
$$

We now follow the usual procedure known as preparing the equation in which we modify the nonlinearity outside the absorbing set $\mathcal{K}^{-1} \mathcal{B}_{2 \kappa\left(U_{1}\right)}$. We modify the nonlinear term:

$$
N_{P}(v, \varphi)= \begin{cases}N(v, \varphi), & \text { if } \mathcal{K} v \in \mathcal{V} \\ 0, & \text { if } \mathcal{K} v \in \mathcal{H} \backslash \mathcal{B}_{2 \kappa\left(U_{1}\right)}\end{cases}
$$

This is clearly a Lipschitz function. Denote by $C>0$ its Lipschitz constant. Following [36], a Lipschitz-continuous function defined on a subset of a Hilbert space can be extended to a Lipschitz continuous function defined on the entire Hilbert space, even preserving the Lipschitz constant $C>0$. Without changing the notation, let us denote such an extension by $N_{P}: \mathcal{H} \times \mathbb{R} \rightarrow \mathbb{R}$. The prepared equation now reads ass

$$
v_{t}=v_{\varphi \varphi}+\frac{G}{2} v_{\varphi}+N_{P}(v, \varphi)
$$

Finally, the traveling wave transformation $w(t, \varphi)=v\left(t, \varphi-\frac{G}{2} t\right)$ leads to the equation

$$
w_{t}=w_{\varphi \varphi}+N_{P}\left(w, \varphi-\frac{G}{2} t\right) .
$$

The term $N_{P}\left(w, \varphi-\frac{G}{2} t\right)$ is now globally Lipschitz, periodic in both $t$ and $\varphi$, and we find ourselves in the situation of Theorem A.1. 
3.4. Main theorem. We are now able to prove the existence of the inertial manifold of the Smoluchowski equation.

Theorem 3.5. Let $U>2$. The Smoluchowski Equation (2.1) possesses in inertial manifold $\mathcal{M}_{U}$.

The positivity of $A w=-w_{\varphi \varphi}$, the global Lipschitz continuity of $N_{P}$ and the fact that it vanishes outside of a ball in $\mathcal{H}$ suffice to prove that the prepared equation has a solution for all positive times for any initial datum in $\mathcal{H}$, and it is dissipative. The complete set of eigenfunctions for the linear operator $A$ is given by $w_{1}^{n}(\varphi)=$ $\cos n \varphi, w_{2}^{n}(\varphi)=\sin n \varphi, n=0,1, \ldots$, with eigenvalues $\lambda_{n}=n^{2}, n=0,1, \ldots$ If $C_{1}$ is a Lipschitz constant for $N_{P}$, there exists $n \in \mathbb{N}$ such that $\lambda_{n+1}-\lambda_{n}=2 n+1>4 C_{1}$, and the spectral gap condition is satisfied. Theorem A.1 applies, and we infer the existence of a nonautonomous time-periodic inertial manifold $\mathcal{M}_{P}$ for the prepared equation (3.13), given as a graph of a Lipschitz function $\Phi_{P}$ :

$$
\mathcal{G}\left[\Phi_{P}\right]=\left\{\left(t, p+\Phi_{P}(t, p)\right): t \in \mathbb{R}, p \in P_{n} \mathcal{H}\right\}
$$

Because of the fact that $\Phi_{P}$ is Lipschitz in both components, it can be easily seen that the set

$$
\mathcal{M}_{P}=\bigcup_{t \in \mathbb{R}}\left(p+\Phi_{P}(t, p)\right)\left(\cdot+\frac{G}{2} t\right)
$$

is an inertial manifold for (3.12). We now define $\mathcal{M}_{U}=B_{\rho_{0}(U)} \cap \Theta^{-1} \mathcal{G}_{U}\left(\mathcal{X}_{U} \cap\right.$ $\mathcal{K}\left(\mathcal{M}_{P}\right)$ ). Since $\mathcal{G}_{U}: \mathcal{X}_{U} \rightarrow X$ is a Lipschitz homeomorphism, it is immediate that $\mathcal{M}_{U}$ is a finite dimensional Lipschitz manifold. It is positively invariant under, since both $B_{\rho_{0}(U)}$ and $\Theta^{-1} \mathcal{G}_{U}\left(\mathcal{X}_{U} \cap \mathcal{K}\left(\mathcal{M}_{P}\right)\right)$ are positively invariant. It is also nonempty, since it contains the global attractor of (3.12). It remains to prove that $\mathcal{M}_{U}$ is exponentially tracking. Let $f_{0} \in H$ and $f(t)=\Sigma(t) f_{0}$. Let $v(t)=\mathcal{K}^{-1} \mathcal{G}_{\Upsilon}^{-1} \Theta(f(t)), t \geq 0$. Since $\mathcal{M}_{P}$ is exponentially tracking, there exists $v_{0} \in \mathcal{M}_{P}$ such that for the solution $v_{P}$ of (3.12) to this initial datum we have $\left\|v(t)-v_{P}(t)\right\|_{L^{2}} \rightarrow 0$, as $t \rightarrow \infty$, exponentially. Also, there exists $T>0$ such that $v_{P}(t) \in \mathcal{K}^{-1}(\mathcal{U})$ for $t \geq T$. However, since $\left.N_{P}\right|_{\mathcal{K}^{-1}(\mathcal{U})}=\left.N\right|_{\mathcal{K}^{-1}(\mathcal{U})}$, $\Upsilon\left(\mathcal{K}\left(v_{P}(t)\right)\right)=U$ for $t \geq T$. Therefore, $h(t):=\Theta^{-1} \mathcal{G} \mathcal{K}\left(v_{P}(t)\right) \in \Theta^{-1} \mathcal{G K}\left(\mathcal{M}_{P}\right), t \geq T$ is a solution of (2.1). For some $T^{\prime} \geq T$ we have $h(t) \in B_{\rho_{0}(U)}, t \geq T^{\prime}$, and therefore $h(t) \in \mathcal{M}_{U}, t \geq T^{\prime}$. Finally, since all the transformations are Lipschitz continuous, $\|f(t)-h(t)\|_{L^{2}} \rightarrow 0$ as $t \rightarrow \infty$, exponentially. This concludes the proof.

Appendix A. Inertial manifolds for nonautonomous evolution equations. In this section, we shall define time-dependent (nonautonomous) inertial manifolds and state and partially prove a theorem on their existence. Just like for the autonomous evolution equations, for which the theory originated, there now exists a well developed theory of inertial manifolds for the nonautonomous evolution equations, which applies to our case. In papers [21, 22, 23], the authors prove the existence using cone invariance and strong squeezing properties. In [2], the authors used the Lyapunov-Perron method to prove the existence of inertial manifolds for the case in which $\mathrm{N}$ is assumed to be linear in $\mathrm{u}$ and periodic in $t$. Following this paper, without reproducing the entire proof, we shall indicate here how the inertial manifolds are constructed using the Lyapunov-Perron method for the case of nonlinear $N$ and periodic $t$. 
We consider an evolution equation on a Hilbert space $H$ endowed with the inner product $(\cdot, \cdot)$, and the norm $|\cdot|$ of the form

$$
\frac{d u}{d t}+A u=N(t, u)
$$

where $A$ is a positive self-adjoint linear operator with compact inverse, and $N: \mathbb{R} \times$ $H \rightarrow H$ is a locally Lipschitz function in $u$ and $T$-periodic and continuous in $t$. Recall that, since $A^{-1}$ is compact, there exists a complete set of eigenfunctions $w_{k}$ for $A$

$$
A w_{k}=\lambda_{k} w_{k}
$$

We arrange the eigenvalues in a nondecreasing sequence $\lambda_{k} \leq \lambda_{k+1}, k=1,2, \ldots$ It is a well known fact that $\lambda_{k} \rightarrow \infty$ as $k \rightarrow \infty$. We also define the projection operators

$$
P_{n} u=\sum_{k=1}^{n}\left(u, w_{k}\right) w_{k}
$$

and $Q_{n}=I-P_{n}$.

For the autonomous case, i.e., when $N(t, u)=N(u)$, we define inertial manifolds in the following way.

Definition A.1. An inertial manifold $\mathcal{M}$ is a finite-dimensional Lipschitz manifold which is positively invariant, i.e.,

$$
\Sigma(t) \mathcal{M} \subset \mathcal{M}, t \geq 0,
$$

and has the exponential tracking property, i.e., there exist $\mu>0$ so that for every $u_{0} \in H$ there exists $v_{0} \in \mathcal{M}$ such that

$$
\left|\Sigma(t) u_{0}-\Sigma(t) v_{0}\right| \leq K e^{-\mu t}, \quad t \geq 0
$$

where $K=K\left(u_{0}, v_{0}\right)>0$.

The dynamics of a a nonautonomous system are no longer described by a semigroup, and thus the definitions for invariant sets, global attractors and inertial manifolds have to be modified. Rather than by a semigroup, the dynamics are described by a two parameter family $\{\Sigma(t, \theta): t \geq \theta \in \mathbb{R}\}$ (or $\Sigma(\cdot, \cdot)$ for short) of continuous operators from $H$ into itself such that

- $\Sigma(\theta, \theta)=I$

- $\Sigma(t, \sigma) \Sigma(\sigma, \theta)=\Sigma(t, \theta)$

- $(t, \theta) \mapsto \Sigma(t, \theta) u_{0}$ is continuous for $t \geq \theta$ and $u_{0} \in H$.

The natural way to define invariance in this context is by the following.

Definition A.2. A family $A=\{A(t)\}_{t \in \mathbb{R}}$ of nonempty sets $A(t) \subset H$ is called a nonautonomous set. It will be said to be forward invariant if

$$
\Sigma(t, \theta) A(\theta) \subset A(t), t \geq \theta
$$

and invariant if

$$
\Sigma(t, \theta) A(\theta)=A(t), t \geq \theta .
$$


In this context, one also talks about two different kinds of attraction (dynamics), the forward $(t \rightarrow \infty)$, and the pullback $(\theta \rightarrow-\infty)$ attraction (dynamics). In general, these two dynamics are different, and they will coincide only in some specific situations, e.g., when $\Sigma(\cdot, \cdot)$ is a semigroup in disguise. Let us recall here that for autonomous systems, the global attractor can be characterized as the union of all global in time defined trajectories which are also bounded. In order to achieve the same classification for nonautonomous systems, it turns out that in the definition of the global attractor we need to require the pullback attraction. We arrive at the following.

Definition A.3. A pullback attractor is defined as an invariant family $A=\{A(t)\}_{t \in \mathbb{R}}$ of compact sets $A(t) \subset H$ such that $\overline{\cup_{t \in \mathbb{R}} A(t)}$ is compact, and it attracts all bounded $B \subset H$ in the pullback sense

$$
\lim _{\theta \rightarrow-\infty} \operatorname{dist}(\Sigma(\mathrm{t}, \theta) \mathrm{B}, \mathrm{A}(\mathrm{t}))=0, \mathrm{t} \in \mathbb{R} .
$$

As for inertial manifolds, the definition has to be modified in the following way:

Definition A.4. A nonautonomous inertial manifold is a nonautonomous set $\mathcal{M}$ with the properties that

- for each $t \in \mathbb{R}, \mathcal{M}(t)$ is a finite-dimensional Lipschitz manifold,

- it is invariant,

- and it has the exponential tracking property, i.e., there exist $\mu>0$ so that for every $\theta \in \mathbb{R}$ and $u_{0} \in H$ there exists $v_{0} \in \mathcal{M}(\theta)$ such that

$$
\left|\Sigma(t, \theta) u_{0}-\Sigma(t, \theta) v_{0}\right| \leq K e^{-\mu t}, \quad t \geq 0
$$

where $K=K\left(\theta, u_{0}, v_{0}\right)>0$.

We shall need the following version of the existence theorem.

THEOREM A.1. Suppose that the nonlinearity $N(t, u)$ in (A.1) satisfies the following three conditions.

- It has compact support in $H$, i.e., $\operatorname{supp}(N(t, \cdot)) \subset B_{\rho}=\{u \in H:|u| \leq \rho\}$ for some $\rho>0$.

- It is continuous in $t$, and $|N(t, u)| \leq C_{0}$ for $t \in \mathbb{R}$ and $u \in H$, and for some constant $C_{0}>0$.

- It is globally Lipschitz continuous, i.e. $\left|N\left(t_{1}, u_{1}\right)-N\left(t_{2}, u_{2}\right)\right| \leq C_{1}\left|u_{1}-u_{2}\right|+$ $C_{2}\left|t_{1}-t_{2}\right|$ for $t_{1}, t_{2} \in \mathbb{R}, u_{1}, u_{2} \in H$, and for some constants $C_{1}, C_{2}>0$.

Suppose that the eigenvalues of $A$ satisfy the spectral gap condition

$$
\lambda_{n+1}-\lambda_{n}>4 C_{1}
$$

for some $n \in \mathbb{N}$. Then there exists a T-periodic Lipschitz continuous function $\Phi$ : $\mathbb{R} \times P_{n} H \rightarrow Q_{n} H$ so that the nonautonomous set given as a graph of $\Phi$

$$
\mathcal{M}=\mathcal{G}[\Phi]=\left\{(t, p+\Phi(t, p)): t \in \mathbb{R}, p \in P_{n} H\right\}
$$

is an inertial manifold. Restricting (A.1) to $\mathcal{M}$ yields the ordinary differential equation for $p=P_{n} u$

$$
\frac{d p}{d t}+A p=P_{n} N(t, p+\Phi(t, p))
$$


termed the inertial form.

(sketch) Let $\theta \in \mathbb{R}$ and consider the equation

$$
\frac{d u}{d t}+A u=N(\theta+t, u)
$$

Let $\beta=\lambda_{n+1}-\lambda_{n}$ and $\eta=\beta / 2$. Define the Banach space

$$
X^{-}=\left\{f: \mathbb{R}^{-} \rightarrow H: f \text { continuous and } \sup _{t \leq 0}\left|e^{\eta t} f(t)\right|<\infty\right\}
$$

First of all, observe that if $u=e^{-\lambda_{n} t} v$, then (A.3) is equivalent to

$$
\frac{d v}{d t}=\left(\lambda_{n}-A\right) v+e^{\lambda_{n} t} N\left(t+\theta, e^{-\lambda_{n} t} v\right)
$$

Next, note that a function $v \in X^{-}$is a solution if and only if it satisfies the integral equation

$$
\begin{aligned}
v(t)= & e^{\left(\lambda_{n}-A\right) t} p+\int_{0}^{t} e^{\left(\lambda_{n}-A\right)(t-s)} P_{n} e^{\lambda_{n} s} N\left(\theta+s, e^{-\lambda_{n} s} v(s)\right) d s \\
& +\int_{-\infty}^{t} e^{\left(\lambda_{n}-A\right)(t-s)} Q_{n} e^{\lambda_{n} s} N\left(\theta+s, e^{-\lambda_{n} s} v(s)\right) d s
\end{aligned}
$$

where $p=P_{n} v(0)$. To see this, first observe that for a solution $v \in X^{-}$and for $\tau \leq t \leq 0$, we have by the variation of constants formula

$$
P_{n} v(t)=e^{\left(\lambda_{n}-A\right) t} p+\int_{0}^{t} e^{\left(\lambda_{n}-A\right)(t-s)} P_{n} e^{\lambda_{n} s} N\left(\theta+s, e^{-\lambda_{n} s} v(s)\right) d s
$$

and

$$
Q_{n} v(t)=e^{\left(\lambda_{n}-A\right)(t-\tau)} Q_{n} v(\tau)+\int_{\tau}^{t} e^{\left(\lambda_{n}-A\right)(t-s)} Q_{n} e^{\lambda_{n} s} N\left(\theta+s, e^{-\lambda_{n} s} v(s)\right) d s
$$

Then, observe that

$$
\left|e^{\left(\lambda_{n}-A\right)(t-\tau)} Q_{n} v(\tau)\right| \leq e^{-\beta t+(\beta-\eta) \tau}|v|_{X^{-}} \rightarrow 0
$$

as $\tau \rightarrow-\infty$. Thus

$$
Q_{n} v(t)=\int_{-\infty}^{t} e^{\left(\lambda_{n}-A\right)(t-s)} Q_{n} e^{\lambda_{n} s} N\left(\theta+s, e^{-\lambda_{n} s} v(s)\right) d s
$$

The converse can also be established using the usual arguments.

Define the function $F$ in the following way:

$$
\begin{aligned}
F: X^{-} \times P_{n} H \times \mathbb{R} & \rightarrow X^{-} \\
(v, p, \theta) \mapsto & e^{\left(\lambda_{n}-A\right) t} p+\int_{0}^{t} e^{\left(\lambda_{n}-A\right)(t-s)} P_{n} e^{\lambda_{n} s} N\left(\theta+s, e^{-\lambda_{n} s} v(s)\right) d s \\
& +\int_{-\infty}^{t} e^{\left(\lambda_{n}-A\right)(t-s)} Q_{n} e^{\lambda_{n} s} N\left(\theta+s, e^{-\lambda_{n} s} v(s)\right) d s .
\end{aligned}
$$


It can be easily observed that $F$ is well defined and that

$$
\begin{aligned}
|F(v, p, \theta)-F(\bar{v}, p, \theta)|_{X^{-}} & \leq C_{1}\left(\frac{1}{\eta}+\frac{1}{\beta-\eta}\right)|v-\bar{v}|_{X^{-}} \\
& \leq \frac{4 C_{1}}{\beta}|v-\bar{v}|_{X^{-}} .
\end{aligned}
$$

The assumption on the spectral gaps insures that $F$ is a contraction with respect to the function $v$, thus for fixed $p \in P_{n} H$ and $\theta \in \mathbb{R} F$ possesses a unique fixed point $v(\cdot, p, \theta) \in X^{-}$. We now define

$$
\begin{aligned}
\Phi(\theta): P_{n} H & \rightarrow Q_{n} H \\
p & \mapsto Q_{n} v(0, p, \theta)=\int_{-\infty}^{0} e^{s A} Q_{n} N\left(\theta+s, e^{-\lambda_{n} s} v(s)\right) d s .
\end{aligned}
$$

The function $\Phi$ is $T$-periodic in $\theta$ and Lipschitz continuous in $p$. To see that, let $v=F(v, p, \theta)$ and $\bar{v}=F(\bar{v}, \bar{p}, \theta)$ for $p$ and $\bar{p} \in P_{n} H$. Then

$$
\begin{aligned}
|v(\cdot, p, \theta)-\bar{v}(\cdot, \bar{p}, \theta)|_{X^{-}} & =|F(v, p, \theta)-F(\bar{v}, \bar{p}, \theta)|_{X^{-}} \\
& \leq \frac{4 C_{1}}{\beta}|v(\cdot, p, \theta)-\bar{v}(\cdot, \bar{p}, \theta)|_{X^{-}}+|p-\bar{p}|
\end{aligned}
$$

so

$$
|v(\cdot, p, \theta)-\bar{v}(\cdot, \bar{p}, \theta)|_{X^{-}} \leq \frac{\beta}{\beta-4 C_{1}}|p-\bar{p}|
$$

and

$$
|\Phi(\theta)(p)-\Phi(\theta)(\bar{p})| \leq \frac{4 C_{1}}{\beta-4 C_{1}}|p-\bar{p}| .
$$

One of the advantages of having an inertial form for the system is that, at least theoretically, we can preform an asymptotic study of the original system via nonlinear Floquet theory. The following theorem can be proven exactly like for its linear counterpart.

TheOREM A.2. Let $F_{0, t}$ be the the flow map generated by the inertial form (A.2), and let $\Psi=F_{0, T}$ be the corresponding monodromy map. If the monodromy map has a logarithm, i.e., if there exists an autonomous vector field $Z$ such that $\Psi=\exp (T Z)$, then there exists T-periodic $P$ so that

$$
F_{0, t}=P(t) \circ \exp (Z t) .
$$

The mapping $P$ is called the Floquet mapping and the eigenvalues of $Z$ are called the Floquet exponents. Finding the monodromy matrix is a nontrivial task, if at all possible. In the chronological calculus formalism we can write

$$
Z(p)=-A p+\frac{1}{T} \ln \overrightarrow{\exp }\left(\int_{0}^{T} P_{n} N(\tau, p+\Phi(\tau, p) d \tau) .\right.
$$

Acknowledgement. This work was supported in part by the NSF grant DMS0733126 and the PSC-CUNY grant PSCOOC-38-125. 


\section{REFERENCES}

[1] R. Bandyopadhyay, G. Basappa and A.K. Sood, Observation of chaos dynamics in dilute sheared aqueous solutions of CTAT, Phys. Rev. Lett., 84, 2022-2025, 2000.

[2] S.N. Chow, K. Lu and J. Mallet-Paret, Floquet theory for parabolic differential equations, J. Diff. Eq., 109, 147-200, 1994.

[3] S.N. Chow, K. Lu and G.R. Sell, Smoothness of inertial manifolds, J. Math. Anal. Appl., 169, 283-312, 1992.

[4] P. Constantin, Smoluchowski Navier-Stokes systems, Contemporary Mathematics, 429, 85-109, 2007.

[5] P. Constantin, C. Foias, B. Nicolaenko and R. Temam, Integral and Inertial Manifolds for Dissipative Partial Differential Equations, Springer-Verlag, Applied Math. Sciences, 70, New York, 1989.

[6] P. Constantin, I. Kevrekidis and E.S. Titi, Remarks on a Smoluchowski equation, Discrete and Continuous Dynamical Systems, 11(1), 101-112, 2004.

[7] P. Constantin, I. Kevrekidis and E.S. Titi, Asymptotic states of a Smoluchowski equation, Arch. Rat. Mech. Anal., 174, 365-384, 2004.

[8] P. Constantin, E.S. Titi and J. Vukadinovic, Dissipativity and Gevrey regularity of a Smoluchowski equation, Indiana Univ. Math. J., 54(44), 949-969, 2005.

[9] P. Constantin and J. Vukadinovic, Note on the number of steady states for a 2D Smoluchowski equation, Nonlinearity, 18, 441-443, 2005.

[10] M. Doi, Molecular dynamics and rheological properties of concentrated solutions of rodlike polymers in isotropic and liquid crystalline phases, J. Polym. Sci., Polym. Phys. Ed., 19, 229-243, 1981.

[11] M. Doi and S.F. Edwards, The Theory of Polymer Dynamics, Oxford University Press (Clarendon), London, New York, 1986.

[12] F. Faraoni, M. Grosso, S. Crescitelli and P.L. Maffetone, The rigid rodmodel for nematic polymers: an analysis of the shear flow problem, J. Rheol., 43(3), 829-843, 1999.

[13] I. Fatkullin and V. Slastikov Critical points of the Onsager functional on a sphere, Nonlinearity, 18, 2565-2580, 2005.

[14] C. Foias, B. Nikolaenko, G.R. Sell and R. Temam, Inertial manifolds for the KuramotoSivashinsky equation and an estimate of their lowest dimension, J. Math. Pures Appl., 67, 197-226, 1988.

[15] C. Foias, G.R. Sell and R. Temam, Variétés inertielles des équations differeéntielles dissipatives, C.R. Acad. Sci. Paris I, 301, 285-288, 1985.

[16] C. Foias, G.R. Sell and R. Temam, Inertial manifolds for nonlinear evolutionary equations, J. Diff. Eq., 73, 309-353, 1988.

[17] G. Forest and Q. Wang, Monodomain response of finite-aspect-ratio macromolecules in shear and related linear flows, Rheol. Acta, 42, 20-46, 2003.

[18] G. Forest, Q. Wang and R. Zhou, The weak shear kinetic phase diagram for nematic polymers, Rheol. Acta, 43, 17-37, 2004.

[19] M. Grosso, R. Keunings, S. Crescitelli and P.L. Maffettone, Prediction of chaotic dynamics in sheared liquid crystalline polymers, Phys. Rev. Lett, 86(14), 3184-3187, 2001.

[20] S.Z. Hess Fokker-Planck-equation approach to flow alignment in liquid crystals, Z. Naturforsch. A, 31, 1034-1037, 1976.

[21] N. Koksch and S. Siegmund, Inertial manifolds for abstract nonautonomous dynamical systems and for nonautonomous evolution equations, Proceedings of the EQUADIFF, 221-266, 2001.

[22] N. Koksch and S. Siegmund, Pullback attracting inertial manifolds for nonautonomous dynamical systems, J. Dynam. Diff. Eqs., 14, 889-941, 2002.

[23] N. Koksch and S. Siegmund, Cone invariance and squeezing properties for inertial manifolds of nonautonomous evolution equations, Banach Center Publications, 60, 27-48, 2002.

[24] R.J. Larson, Arrested tumbling in shearing flows of liquid crystal polymers, Macromolecules, 23, 3983-3992, 1990.

[25] H. Liu, H. Zhang and P. Zhang, Axial symmetry and classification of stationary solutions of Doi-Onsager equation on the sphere with Maier-Saupe potential, Commun. Math. Sci., 3(2), 201-218, 2005.

[26] C. Luo, H. Zhang and P. Zhang, The structure of equilibrium solutions of one dimensional Doi equation, Nonlinearity, 18, 379-389, 2005.

[27] P.L. Maffettone and S. Crescitelli, Bifurcation analysis of a molecular model for nematic polymers in shear flows, J. Non-Newtonian Fluid Mech., 59, 73-91, 1995.

[28] J. Mallet-Paret and G.R. Sell, Inertial manifolds for reaction diffusion equations in higher 
space dimensions, J. Amer. Math. Soc., 1, 805-866, 1988.

[29] W. Maier and A. Saupe, Eine einfache molekular-statistische Theorie der nematischen kristallinflüssigen Phase, Tail I, Z. Naturforsch. A, 14, 882-889, 1959.

[30] G. Marrucci and P.L. Maffettone, Description of the liquid-crystalline phase of rodlike polymers at high shear rates, Macromolecules, 22, 4076-4082, 1989.

[31] L. Onsager, The effects of shape on the interaction of colloidal particles, Ann. N. Y. Acad. Sci., 51, 627-659, 1949.

[32] J.C. Robinson, Inertial manifolds and the cone condition, Dyn. Sys. Appl., 2, 311-330, 1993.

[33] J.C. Robinson, A concise proof of the geometric construction of inertial manifolds, Physics Letters A, 200, 415-417, 1995.

[34] J. Vukadinovic, Inertial manifolds for a Smoluchowski equation on a circle, Nonlinearity, 21, 1533-1545, 2008.

[35] J. Vukadinovic, Inertial manifolds for a Smoluchowski equation on the unit sphere, Commun. Math. Phys., to appear, 2008.

[36] J.H. Wells and L.R. Williams, Embeddings and Extensions in Analysis, Ergebnisse der Mathematik und ihrer Grenzgebiete, Springer Verlag, New York-Hedelberg, 1975. 